\title{
Factores asociados con el uso inconsistente de condón en hombres que tienen sexo con hombres de Ciudad Juárez
}

Factors associated with infrequent condom use amongst men having sex with other men in Ciudad Juárez

Juan C. Mendoza-Pérez ${ }^{1}$ y Luis Ortiz-Hernández ${ }^{2}$

1 Programa Compañeros A.C., Ciudad Juárez, Chihuahua. México

2 Departamento de Atención a la Salud. Universidad Autónoma Metropolitana Xochimilco. México.1ortiz@correo.xoc.uam.mx.

Recibido 18 Marzo 2009/Enviado para Modificación 6 Septiembre 2009/Aceptado 17 Septiembre 2009

\section{RESUMEN}

Objetivo Analizar los factores asociados con el uso inconsistente de condón en hombres que tienen sexo con hombres $(\mathrm{HSH})$ de Ciudad Juárez, Chihuahua.

Material y métodos Se realizó un estudio observacional, transversal y analítico con una muestra por conveniencia $(n=186)$ de HSH de Ciudad Juárez. Las variables independientes fueron: nivel socioeconómico, actitudes hacia del uso del condón, uso de drogas, consumo de alcohol, identidad por orientación sexual y la discriminación por orientación sexual. Las prácticas sexuales que se relacionan con la infección por VIH (uso inconsistente de condón y recibir semen en boca o ano) fueron consideradas como variables dependientes.

Resultados. El 30,0 \% de los HSH entrevistado usaba condón de manera inconsistente cuando practicaban sexo anal receptivo, mientras que la proporción fue de 39,1\% cuando tenían sexo anal insertivo; y el 35,5\% reportaron que recibían semen en la boca 0 ano. Mediante modelos de regresión logística multivariada se observó que los siguientes grupos tuvieron mayor probabilidad de mantener alguna práctica sexual de riesgo: los sujetos de menor edad, con ingreso medio, que presentan actitudes negativas hacia el uso del condón, que consumen drogas o alcohol y que asumían una identidad homosexual.

Conclusiones. Una proporción importante de los HSH entrevistados de la Ciudad Juárez tiene uso inconsistente de condón. Se discuten las implicaciones que tienen los hallazgos en el diseño de programas encaminados a promover el uso de condón entre los $\mathrm{HSH}$.

Palabras Clave: Condones, México, VIH, homosexualidad, bisexualidad (fuente: DeCS, BIREME).

\section{ABSTRACT}

Objective Analysing the factors associated with inconsistent condom use amongst men having sex with other men (MSM) in Ciudad Juárez, Chihuahua. 
Materials and methods This was an observational, cross-sectional analytic study using a convenience sample $(n=186)$ of MSM from Ciudad Juarez. Socioeconomic status, attitudes toward condom use, drug use, alcohol consumption, sexual orientation were considered as independent variables. Dependent variables were sexual behaviour related to HIV infection (inconsistent condom use in anal penetration and receiving semen in the mouth or anus).

Results Whereas $30 \%$ of the MSM interviewed infrequently used a condom when they practiced receptive anal sex, when they had insertive anal sex, $39.1 \%$ reported receiving semen in the mouth and $35.5 \%$ in the anus. Multivariate logistic regression models were used, observing that the groups of men having the highest probability of risky sexual behavior (compared to their counterparts) were younger men ( $\leq 20$ years), those earning medium income, those having a negative attitude towards condom use, men using drugs or alcohol and those who reported homosexual identity. Conclusions An important percentage of MSM infrequently used a condom. The implications of our findings should be used when designing programmes for promoting condom use amongst MSM.

Key Words: Condoms, Mexico, HIV, homosexuality, bisexuality (source: MeSH, NLM).

A finales del 2006, ONUSIDA estimó que a nivel mundial existen 39,5 millones de personas que viven con el virus de la inmunodeficiencia humana (VIH), de los cuales 37,2 millones eran adultos, 17,7 millones mujeres y 2,3 millones menores de 15 años (1). En México, para el 2006 la prevalencia de VIH en población adulta de 15 a 49 años de edad fue de $0,3 \%$, lo que implica que existían 182 mil personas infectadas (1). Según las categorías de transmisión, 92,3\% de los casos se han originado por transmisión sexual, los cuales se distribuyen entre hombres que tienen sexo con hombres (HSH) $(46,7 \%)$ y conducta heterosexual $(45,6 \%)(2)$.

De los casos acumulados de VIH/SIDA entre 1984 al 2005 en el estado de Chihuahua, el 80,3\% de los casos ocurrieron en sujetos de 15 a 44 años de edad (3). En el primer semestre del 2005 en Chihuahua se reportaron 165 casos nuevos, de los cuales 70,0 \% fueron hombres entre 15 y 40 años, dentro de este el 80,0 \% fueron $\mathrm{HSH}(4)$.

$\mathrm{Al}$ inicio de la epidemia de VIH la mayoría de los casos se concentraron en HSH. Las estadísticas previas señalan que si bien la transmisión heterosexual ha comenzado a ser una causa importante, los HSH representan aún un grupo con un alto riesgo. El mayor riesgo de infección en $\mathrm{HSH}$ puede atribuirse a la mayor susceptibilidad biológica derivada de ciertas prácticas sexuales, por ejemplo, la mucosa anal con mayor facilidad puede lesionarse durante la penetración. Sin embargo, algunos autores han llamado la atención respecto al papel de la 
vulnerabilidad social en la concentración de la epidemia en ciertos grupos sociales (5). En el caso de los HSH, la vulnerabilidad social es derivada del estigma asociado con sus prácticas sexuales así como las experiencias de prejuicio, discriminación y violencia que con frecuencia experimentan.

Las prácticas sexuales son de gran interés en las investigaciones sobre VIH/ SIDA, ya que el contacto sexual es el principal mecanismo de transmisión de la infección entre varones. Lo anterior pone de relieve la importancia de estudiar los factores que inciden en el uso o no del condón y los comportamientos asociados con mayor riesgo para la infección por el VIH, como es el uso de sustancias (6). Sin embargo, es necesario precisar que la infección se origina por el contacto con el virus y no por las prácticas sexuales en sí mismas. La cuestión que se plantea como indispensable entonces, es avanzar en la investigación de aquellos elementos socioculturales que determinan que una persona tome o no medidas preventivas eficaces, como el uso del condón (7).

Existen investigaciones en las que se ha analizado la dinámica de la infección en población heterosexual (8-13). De los estudios realizados en México acerca de la infección de VIH/SIDA en la población HSH, una proporción importante se han enfocado en determinar la prevalencia de la infección en este grupo $(9$, 14-17). Sin embargo, existen menos estudios que analizan los factores asociados al uso del condón en $\operatorname{HSH}(7,14,15,18)$.

Los estudios sobre el uso del condón en HSH que radican en ciudades fronterizas son escasos (7), y algunos se realizaron una (18) o dos décadas atrás (15). La escasez de investigaciones sobre esta temática probablemente se debe al estigma asociado a la homosexualidad, lo que contribuye a una invisivilización de este problema de salud pública. Las ciudades fronterizas con Estados Unidos tienen ciertas características que no presentan otras ciudades y que pueden asociarse con el uso de condón. La prevalencia del uso de drogas ilegales es mayor en las ciudades fronterizas que en otras áreas del país (19). Estas ciudades presentan una dinámica de migración y movilidad de la población que hace que muchas personas no cuenten con su red social de origen. La proximidad con Estados Unidos de Norteamérica propicia la interacción sexual y no sexual entre HSH de ambos países. Finalmente la centralización que aún caracteriza a México hace que algunos programas de promoción del uso del condón no tengan una cobertura óptima en estas ciudades.

Para el desarrollo de programas de prevención de la infección por VIH se requiere conocer los factores que inciden en el uso del condón. Se puede reconocer 
que la infección tiene una dinámica multicausal, por lo que es necesario reconocer que las características del huésped (HSH) y del ambiente (vulnerabilidad social) no son estáticos. Además, es indispensable identificar tanto los factores individuales como los procesos socioculturales que inciden en las conductas. Por lo expuesto, el objetivo de esta investigación fue analizar los factores asociados con el uso inconsistente de condón en HSH de Ciudad Juárez, Chihuahua.

\section{MATERIAL Y MÉTODOS}

Se realizó un estudio observacional, transversal y analítico. El grupo de estudio de la investigación fueron los HSH que residen en Ciudad Juárez. Debido a que no se cuenta con un marco muestral para esta población, la muestra seleccionada fue por conveniencia. A los HSH les fue aplicado un cuestionario de autoaplicación, a través del Programa Compañeros, A. C., una organización no gubernamental enfocada a la prevención de VIH. Con el programa EPIDAT, es estimó que se requería por lo menos una muestra de 179 varones. Este tamaño de muestra se calculó considerando los resultados de un estudio previo (7), en el cual se reportó una prevalencia de uso inconsistente de condón de $35 \%$ y se definió un margen de error de $7 \%$ y un nivel de confianza del $95 \%$. Al término del trabajo de campo fueron encuestados 186 varones $\mathrm{HSH}$.

El trabajo de campo fue llevado a cabo de noviembre del 2006 a marzo del 2007, En todos los casos se obtuvo el consentimiento informado por escrito de los participantes. Como variables independientes se tomaron en cuenta los reactivos de las secciones de datos socioeconómicos, actitudes hacia el uso del condón, uso de drogas, consumo de alcohol, identidad por orientación sexual y la discriminación contenidos en la encuesta. En la sección de datos socioeconómicos se conformaron tres grupos para la clasificación de la edad: a) de 20 años y menos, b) de 21 a 29 años, y c) de 30 años y más. El ingreso mensual fue agrupado en tres estratos: 1) de 3999 pesos mexicanos (equivalente a 372.0 dólares americanos) y menos, 2) 4000 a 9999 pesos (372.1 a 930.1 dólares), y 3) 10000 (930.2 dólares) o más. A partir de la escolaridad se formaron tres grupos: 1) hasta secundaria, 2) bachillerato y 3 ) superior y más.

Las actitudes hacia el uso del condón se indagaron con tres indicadores construidos a partir de frases sobre las opiniones que tenían los entrevistados: 1) sabe usar adecuadamente el condón (marcaba positivo la frase "considera que no sabe usarlo adecuadamente"), 2) actitud positiva hacia el condón (elegían la opción "le gusta usarlo" y 3) actitud negativa hacia el condón (seleccionaban las opciones "no le agrada usarlo", "le da igual usarlo ono usarlo" y "no le gusta usarlo"). 
Se indagó el uso de drogas formándose los siguientes grupos: 1) ha probado marihuana, 2) ha probado inhalantes, 3) ha probado cocaína, crack y heroína y 4) ha probado drogas sintéticas (anfetaminas y metanfetaminas). A partir de las preguntas anteriores se formó una nueva variable sobre el uso de alguna droga. El consumo de alcohol en el último mes se evaluó en tres categorías: 1) no consume alcohol, 2) al menos una vez a la semana y 3) más de dos veces a la semana, que incluye la opción del consumo diario de alcohol.

Para indagar la identidad por orientación sexual a los entrevistados se les preguntó "Respecto a su orientación sexual ¿Usted se considera?", las opciones de respuesta fueron agrupadas en dos grupos: 1) "heterosexual, le gustan las personas del sexo opuesto" y 2) "homosexual, le gustan las personas de su mismo sexo" y "bisexual, le gustan los hombres y las mujeres", aunque en el último caso solo se ubicó un sujeto. También se preguntó si habían experimentado las siguientes formas de discriminación por orientación sexual: "¿Alguna vez ha recibido algún tipo de maltrato en los servicios de salud por su orientación sexual?", "Cuando recibe servicios médicos, ¿Ha considerado necesario ocultar su orientación sexual?", "¿Le ha sido necesario fingir o decir explícitamente que es heterosexual cuando recibe servicios médicos?" y 4) "¿Ha acudido a solicitar apoyo por algún hecho discriminatorio?".

Las prácticas sexuales que se relacionan con la infección por VIH fueron consideradas como variables dependientes. Se preguntó la frecuencia con la que utilizaban condón cuando tenían las siguientes prácticas: sexo anal insertivo, sexo anal receptivo y sexo oral insertivo. Se consideró que existía uso inconsistente de condón cuando respondían "a veces" y "nunca", mientras que la respuesta "siempre" fue clasificada como uso consistente. También se indagó si recibían semen en la boca o ano, en este caso las opciones "siempre" y "a veces" fueron consideradas indicativas de práctica de riesgo.

Para cada una de las variables se estimaron las estadísticas descriptivas adecuadas (frecuencia relativa). Para conocer si existían diferencias entre los sub-grupos de HSH respecto al uso de condón se estimó la prueba de Chi cuadrado (variables ordinales y nominales). Para estudiar la asociación de las variables independientes con el uso inconsistente de condón se calcularon razones de momios (OR) con sus respectivos intervalos de confianza al $95 \%$. Los OR fueron estimados mediante modelos de regresión logística multivariada, en los cuales primero se introdujeron todas las variables que en el análisis bivariado se habían asociado con el uso inconsistente de condón. En estos modelos se fueron descartando las variables que no se relacionaban con el uso inconsistente de condón ( $p>0,10)$. 


\section{RESULTADOS}

En la Tabla 1 se presenta la distribución de los varones HSH de acuerdo a sus prácticas sexuales. La práctica sexual de menor frecuencia fue la penetración vaginal: 46,8 \% respondió que no aplicaba a su situación. La mayoría de los sujetos que practicaban sexo anal receptivo o insertivo siempre utilizaban condón (60,7 \% y 52,2\%, respectivamente); por el contrario, la mayoría de los HSH que practicaban sexo oral receptivo o insertivo nunca lo utilizaban (57,6\% y 55,3\%, respectivamente). Más de la mitad $(58,4 \%)$ de los entrevistados reportaron que nunca recibían semen en la boca o ano.

La edad promedio de los HSH fue de 25 años, la mayoría contaba con una escolaridad de superior y más; el ingreso mensual que predominó fue de 3999 pesos o menos (372.0 dólares o menos, 47,5 \%). En relación con las actitudes hacia el uso del condón, la mayoría de los HSH reportaron que sabían utilizarlo $(94,0 \%)$, que les gustaba utilizarlo $(91,8 \%)$ y que tenían actitud positiva hacia él $(81,0 \%)$. Respecto al uso de sustancias, casi la mitad de los encuestados había consumido drogas $(42,5 \%)$, siendo la más utilizada la marihuana (36,9\%), seguida por cocaína, crack y heroína $(25,3 \%)$ y las drogas sintéticas $(14,5 \%)$. La mitad de población $(53,9 \%)$ respondió consumir alcohol al menos una vez a la semana. La mayoría de los encuestados se identificaron como homosexuales $(76,7 \%)$. Con relación a la discriminación por orientación sexual, la mayor parte de la población reportó que no había sido maltratada en los servicios de salud $(80,1 \%)$, tampoco ocultaban su orientación cuando recibían atención médica $(76,8 \%)$, ni fingían ser heterosexuales cuando recibían atención médica $(81,1 \%)$. El 6,1 \% había solicitado apoyo por algún acto de discriminación.

En la Tabla 2 se presenta la asociación del uso inconsistente de condón con las características sociodemográficas, las actitudes hacia el condón, el uso de drogas y alcohol, orientación sexual y las experiencias de discriminación. El aumento de la edad se relacionó con menor frecuencia de uso inconsistente de condón cuando tuvieron penetración anal receptiva, sin embargo las diferencias fueron marginalmente significativas $(\mathrm{p}=0,079)$. Con respecto a los sujetos de mayor ingreso, entre los de menor ingreso fue más probable el uso inconsistente de condón en la penetración vaginal. En comparación con las personas mayor ingreso, en las de ingreso medio fue más alta la prevalencia de uso inconsistente de condón en penetración anal receptiva, siendo las diferencias estadísticamente significativas. Respecto a los sujetos que les gustaba utilizar condón, en los que no les gustaba fue más alta la prevalencia de uso inconsistente de condón cuando practicaban penetración anal receptiva o insertiva y que recibieran semen en el 
ano o la boca. Los sujetos con actitud negativa hacia el condón con mayor frecuencia reportaron uso inconsistente de condón cuando practicaban penetración anal receptiva, penetración anal insertiva, sexo oral receptivo y que recibían semen en la boca o ano.

Tabla 1. Frecuencias sobre el uso del condón en las prácticas sexuales

\begin{tabular}{|c|c|c|c|c|c|c|}
\hline & $\mathrm{N}$ & $\begin{array}{l}\mathrm{S} \\
\%\end{array}$ & $\begin{array}{r}\mathrm{AV} \\
\%\end{array}$ & $\begin{array}{r}\mathrm{Nu} \\
\%\end{array}$ & $\begin{array}{r}\text { NA } \\
\%\end{array}$ & $\begin{array}{r}\text { NDC } \\
\%\end{array}$ \\
\hline Penetración vaginal & 126 & 18,3 & 5,6 & 7,9 & 46,8 & 21,4 \\
\hline Penetración anal receptiva & 150 & 60,7 & 23,3 & 6,7 & 0,7 & 8,7 \\
\hline Penetración anal insertiva & 161 & 52,2 & 29,8 & 9,3 & 1,2 & 7,5 \\
\hline Sexo oral receptivo & 165 & 11,5 & 23,6 & 57,6 & 0,6 & 6,7 \\
\hline Sexo oral insertivo & 161 & 12,4 & 25,5 & 55,3 & 0,6 & 6,2 \\
\hline Recibir semen en la boca o ano & 166 & 3,6 & 31,9 & 58,4 & 0,6 & 5,4 \\
\hline
\end{tabular}

En comparación con los sujetos que no consumen drogas, entre los sujetos que las han consumido fue más frecuente el uso inconsistente de condón cuando practicaban penetración anal receptiva, sexo oral receptivo, sexo oral insertivo, además de que con mayor frecuencia recibían semen en ano o boca. Con respecto a los sujetos que no han consumido marihuana, entre los sujetos que la consumieron fue más frecuente el uso inconsistente de condón en penetración anal receptiva y en sexo oral insertivo. Los sujetos que han consumido inhalantes presentaron mayor probabilidad de recibir semen en ano o boca, sin embargo, las diferencias fueron marginalmente significativas $(\mathrm{p}=0,068)$. Entre los participantes del estudio que consumieron cocaína, crack y heroína fue mayor el porcentaje de uso inconsistente de condón en el sexo oral receptivo e insertivo. Las prevalencias de uso inconsistente de condón en el sexo oral insertivo y recibir semen en ano o boca fueron más altas en los usuarios de drogas sintéticas, aunque en el primer caso las diferencias fueron marginales. En los sujetos que consumían alcohol más de dos veces a la semana fue más alto el porcentaje de uso inconsistente de condón en sexo oral receptivo.

En comparación con los varones que mencionaron ser heterosexuales, entre los que indicaron que eran homosexuales fue más frecuente el uso inconsistente de condón en sexo oral receptivo e insertivo y que recibieran semen en ano o boca. Los HSH que habían solicitado apoyo cuando fueron discriminados presentan mayor frecuencia de recibir semen en la boca o ano. 
Tabla 2. Asociación de factores socioeconómicos y actitudes hacia el condón con el uso inconsistente de condón

\begin{tabular}{|c|c|c|c|c|c|c|c|c|c|c|}
\hline & $\mathrm{N}$ & $\%$ & $\begin{array}{l}\text { UIC- } p \\
\text { PV }\end{array}$ & $\begin{array}{ll}\text { UIC- } & p \\
\text { PAR } & \\
\end{array}$ & $\begin{array}{l}\text { UIC- } \\
\text { PAI }\end{array}$ & $p$ & $\begin{array}{l}\text { UIC- } \\
\text { SOR }\end{array}$ & $p$ & $\begin{array}{l}\text { UIC- } p \\
\text { SOI }\end{array}$ & $\begin{array}{ll}\text { RS- } & p \\
\text { BA } & \\
\end{array}$ \\
\hline & & & $\%$ & $\%$ & $\%$ & & $\%$ & & $\%$ & $\%$ \\
\hline \multicolumn{11}{|l|}{ Ingreso (pesos) } \\
\hline 3999 o menos & 75 & 47,5 & $57,1^{*}$ & 23,6 * & 39,0 & & 83,6 & & 82,3 & 32,8 \\
\hline 4000 a 9999 & 57 & 36,1 & 40,0 & 43,2 & 47,8 & & 92,0 & & 91,8 & 50,0 \\
\hline 10000 o más & 26 & 16,5 & 0,0 & 21,1 & 31,8 & & 87,5 & & 86,4 & 29,2 \\
\hline \multicolumn{11}{|c|}{ Le gusta utilizar condón } \\
\hline No & 15 & 8,2 & 58,3 & 46,9 * & 63,9 & * & 91,7 & & 91,2 & $59,5 * *$ \\
\hline Sí & 169 & 91,8 & 35,7 & 29,1 & 36,4 & & 87,0 & & 86,0 & 31,6 \\
\hline \multicolumn{11}{|c|}{ Actitud hacia uso de condón } \\
\hline Negativa & 35 & 19,0 & 50,0 & $57,7^{\star \star}$ & 75,9 & 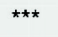 & 93,5 & * & $96,4^{*}$ & $67,7^{\star \star \star}$ \\
\hline Positiva & 149 & 81,0 & 40,0 & 27,5 & 35,0 & & 86,7 & & 85,0 & 30,9 \\
\hline \multicolumn{11}{|c|}{ Ha probado alguna droga } \\
\hline Si & 79 & 42,5 & 46,7 & 40,6 * & 49,3 & & 93,0 & * & 92,8 * & 45,8 * \\
\hline No & 107 & 57,5 & 40,0 & 26,4 & 37,2 & & 82,9 & & 81,5 & 31,0 \\
\hline \multicolumn{11}{|c|}{ Ha probado marihuana } \\
\hline Sí & 63 & 33,9 & 50,0 & $46,0 * *$ & 50,9 & & 91,2 & & 94,5 * & 42,4 \\
\hline No & 123 & 66,1 & 60,0 & 25,6 & 37,8 & & 85,4 & & 82,1 & 35,1 \\
\hline \multicolumn{11}{|c|}{ Cocaína, crack o heroína } \\
\hline Sí & 47 & 25,3 & 45,5 & 31,7 & 46,3 & & 100,0 & $* *$ & 95,2 * & 45,2 \\
\hline No & 139 & 74,7 & 41,4 & 33,7 & 41,5 & & 82,9 & & 83,3 & 35,1 \\
\hline \multicolumn{11}{|c|}{ Drogas sintéticas } \\
\hline Sí & 27 & 14,5 & 40,0 & 36,0 & 44,0 & & 96,0 & & 100,0 * & 54,2 \\
\hline No & 159 & 85,5 & 43,3 & 32,4 & 42,6 & & 85,9 & & 84,0 & 34,8 \\
\hline \multicolumn{11}{|c|}{ Consumo de alcohol } \\
\hline No & 25 & 16,4 & 66,7 & 35,0 & 38,1 & & 71,4 & $* * *$ & 76,2 & 40,9 \\
\hline $\begin{array}{l}\text { Al menos } 1 \mathrm{vez} \\
\text { /semana }\end{array}$ & 82 & 53,9 & 31,3 & 33,3 & 40,8 & & 93,1 & & 90,1 & 33,3 \\
\hline $\begin{array}{l}\text { Más de } 2 \\
\text { veces /semana }\end{array}$ & 45 & 29,6 & 46,2 & 34,2 & 40,5 & & 95 & & 89,7 & 48,8 \\
\hline \multicolumn{11}{|c|}{ Orientación sexual por identidad } \\
\hline Homosexuales & 135 & 76,7 & 42,4 & 32,1 & 43,8 & & 90,5 & * & 89,3 * & $42,4 * *$ \\
\hline Heterosexuales & 41 & 23,3 & 42,9 & 38,1 & 39,1 & & 76,0 & & 72,0 & 17,9 \\
\hline \multicolumn{11}{|c|}{ Solicitó apoyo por discriminación } \\
\hline Sí & 10 & 6,1 & 0,0 & 44,4 & 50,0 & & 87,5 & & 87,5 & 75,0 * \\
\hline No & 155 & 83,3 & 50,0 & 32,5 & 41,9 & & 88,7 & & 87,0 & 36,3 \\
\hline
\end{tabular}

En el uso inconsistente de condón no existieron diferencias de acuerdo a edad, escolaridad, el saber utilizar el condón, haber probado inhalantes, haber sido maltratado en los servicios de salud, ocultar la orientación cuando recibe atención médica o fingir ser heterosexual cuando recibían atención médica (datos no mostrados en tablas). 
Tabla 3. Modelos de regresión logística multivariada teniendo como variable dependiente el uso inconsistente de condón

\begin{tabular}{|c|c|c|c|c|}
\hline & OR & IC $95 \%$ & $x^{2}$ & $p$ \\
\hline \multicolumn{5}{|l|}{ Variable dependiente: UIC-PAR } \\
\hline Edad (años) & & & 5,45 & 0,066 \\
\hline$\leq 20$ & 3,07 & $0,81-11,60$ & 2,74 & 0,098 \\
\hline $21-29$ & 0,82 & $0,26-2,57$ & 0,12 & 0,731 \\
\hline$\geq 30$ & 1,00 & & & \\
\hline Ingreso (pesos) & & & 5,55 & 0,062 \\
\hline$<3999$ & 0,84 & $0,21-3,40$ & 0,06 & 0,805 \\
\hline $4000-9999$ & 2,66 & $0,68-10,40$ & 1,98 & 0,159 \\
\hline$\geq 10000$ & 1,00 & & & \\
\hline Actitud negativa hacia el condón & 2,96 & $1,09-7,99$ & 4,56 & 0,033 \\
\hline \multicolumn{5}{|l|}{ Variable dependiente: UIC- PAI } \\
\hline Edad (años) & & & 6,66 & 0,036 \\
\hline$\leq 20$ & 4,72 & $1,45-15,34$ & 6,65 & 0,010 \\
\hline $21-29$ & 2,48 & $0,92-6,68$ & 3,25 & 0,072 \\
\hline$\geq 30$ & 1,00 & & & \\
\hline Actitud negativa hacia el condón & 6,96 & $2,59-18,69$ & 14,85 & 0,000 \\
\hline \multicolumn{5}{|l|}{ Variable dependiente: UIC- SOR } \\
\hline Consumo de alcohol & & & 8,38 & 0,015 \\
\hline Al menos una vez a la semana & 5,36 & $1,44-19,91$ & 6,29 & 0,012 \\
\hline Más de dos veces a la semana & 7,60 & $1,38-41,95$ & 5,41 & 0,020 \\
\hline No consume al alcohol & 1,00 & & & \\
\hline \multicolumn{5}{|l|}{ Variable dependiente: UIC-SOI } \\
\hline Uso de Drogas & 2,80 & $0,94-8,27$ & 3,45 & 0,063 \\
\hline Homosexual (vs heterosexual) & 3,18 & $1,09-9,21$ & 4,52 & 0,034 \\
\hline \multicolumn{5}{|l|}{ Variable dependiente: RS-BA } \\
\hline Actitud negativa hacia el condón & 4,21 & $1,78-9,93$ & 10,77 & 0,001 \\
\hline Homosexual (vs heterosexual) & 2,86 & $0,99-8,23$ & 3,80 & 0,051 \\
\hline
\end{tabular}

Abreviaturas: UIC, uso inconsistente de condón; PAR, penetración anal receptiva.; PAI, penetración anal insertiva; SOR, sexo oral receptivo; SOI, sexo oral insertivo; RS-BA, recibir semen en boca o ano. Para las variables PAR, PAI, SOR y SOI $0=$ siempre, $1=$ nunca y a veces; en la variable RS-BA, $0=$ nunca, $1=$ siempre $y$ a veces.

\section{DISCUSIÓN}

En los HSH de Ciudad Juárez el uso inconsistente de condón fue de 33,1 \% en el sexo anal receptivo y el 33,9 \% en el sexo anal insertivo, además de que la mayoría reportó inconsistencia en el uso del condón en la práctica de sexo oral receptivo $(87,6 \%)$ e insertivo $(86,7 \%)$. En otros estudios se ha observado que una proporción importante de HSH de México aún mantiene prácticas sexuales asociadas con la infección por VIH. En una muestra de varones HSH de la Ciudad de México se observó que el 37,2 \% y 35,8 \% de los que habían tenido sexo anal insertivo o receptivo, respectivamente, no habían utilizado el preservativo de forma consistente (7). En otra muestra de HSH de la ciudad de México (14) se observó que el $67,0 \%$ de los varones nunca utilizaba condón cuando practicaba sexo insertivo, mientras que cuando se trababa de sexo receptivo el $67,0 \%$ de 
los entrevistados nunca lo utilizaba. En otro estudio (20) con varones HSH de Tijuana se observó que entre los HSH seropositivos para VIH el 18,5\% siempre lo habían usado y en los seronegativos el 26,7 \% siempre lo habían utilizado. El 70,0 \% de HSH de seis ciudades de México (Distrito Federal, Guadalajara, Monterrey, Acapulco, Mérida y Tijuana) no habían utilizado condón en su última relación sexual (15). Con relación al consumo de semen en un estudio (21) se reportó que un poco más del 10,0 \% de los entrevistados reportaron haber consumido semen con una frecuencia de "siempre" y "casi siempre", mientras que el 43,0 \% de los entrevistados "casi nunca" lo habían hecho; en otra muestra de HSH de la ciudad de México (7) se encontró que cerca del 25,0 \% habían realizado esta práctica en alguna ocasión. En los HSH de Ciudad Juárez poco más de un tercio reportó que habían recibido semen en la boca o ano.

Además de la frecuencia de prácticas sexuales de riesgo, el estudio con los HSH de Ciudad Juárez permitió identificar algunos factores asociados con el uso inconsistente de condón. En relación a la edad, en el presente estudio fue observado que el grupo de menor edad presentó mayor riesgo de uso inconsistente de condón en la penetración anal receptiva e insertiva. En HSH de Tailandia (22) se observó que los sujetos de 25 a 29 años de edad tuvieron menor riesgo de sexo anal sin protección en comparación con los sujetos de 18 a 24 años. Sin embargo, en otros estudios con HSH de EUA $(23,24)$ y Ecuador (25) no se existieron diferencias significativas en el uso del condón de acuerdo a la edad. La asociación de la edad puede ser explicada a la experiencia, la etapa del desarrollo biológico en la que se encuentran y factores psicosociales. Parte de los jóvenes tienen escaso acceso a los servicios de atención de la salud o reciben información sobre el VIH/SIDA en un momento de sus vidas en que la algunos comienzan a mantener relaciones sexuales por lo que los mimos no se consideran en una situación de riesgo.

En HSH de Ecuador (25) se encontró que el nivel socioeconómico medio alto y alto se asociaron con el uso del condón. Se ha propuesto que los estratos bajos tienen mayor riesgo de contagio por $\mathrm{VIH}$ (16) debido a las limitadas oportunidades económicas y educativas que tienen, lo que favorece el surgimiento de normas de socialización que conducen a comportamientos de riesgo. El hecho de que los HSH de estrato socioeconómico medio de Ciudad Juárez sean los que tuvieron mayor riesgo de uso inconsistente de condón en la penetración anal receptiva puede ser resultado de que tienen más acceso a espacios comerciales en los que se facilitan o son preámbulo para las relaciones sexuales, tales como baños de vapor, discotecas, bares, centros comerciales, etc., además de que pueden hacer uso con mayor frecuencia de la Internet como un medio para establecer contactos. 
En los HSH de Ciudad Juárez se observó que el consumo de alcohol y el uso de drogas aumentaban el riesgo de uso inconsistente de condón en el sexo oral receptivo e insertivo. En otros estudios con HSH de Estados Unidos de América (EUA) se ha observado que el consumo de alcohol $(24,26)$, drogas (27) o ambos (23) durante las relaciones sexuales está asociado con sexo anal receptivo sin condón. Sin embargo, en HSH de Ecuador (25) y de Tailandia (22) no fueron encontradas diferencias significativas entre los consumidores y no consumidores de alcohol en el uso de condón cuando tenían sexo anal receptivo.

Los HSH de Ciudad Juárez que tenían actitudes negativas hacia el uso de condón presentaron mayor riesgo de recibir semen en ano o boca y de uso inconsistente de condón cuando practicaban penetración anal receptiva o insertiva. En otras muestras de HSH de México (18) y EUA (24) se ha observado el mismo patrón. Esto puede relacionarse en que la actitud emerge como moduladora de las conductas de los sujetos y por consecuencia una visión negativa favorece las conductas de riesgo.

Respecto a los HSH que reportaron ser heterosexuales, en los que se consideraron homosexuales existió mayor riesgo del uso inconsistente del condón en el sexo oral insertivo y de recibir semen en ano o boca. De manera similar, con población de la ciudad de México se reportó que los bisexuales con menos frecuencia practican sexo receptivo sin condón respecto a los homosexuales (28). Desde el inicio de la epidemia de VIH/SIDA se hizo evidente que una proporción importante de varones, a pesar de mantener prácticas sexuales con otros varones, no se identifican como homosexuales. Este grupo ha sido motivo de estudio pues se especula que es un factor que contribuye al incremento de casos de infección en las mujeres heterosexuales (28). Es probable que los varones bisexuales (i.e. tienen relaciones sexuales con hombres y mujeres) tengan con menos frecuencia prácticas sexuales de riesgo para mantener ocultas sus relaciones homosexuales, pues pueden tener conciencia del riesgo de infección para sus parejas mujeres. Por el contrario, los varones que se asumen como homosexuales pueden enfrentarse a diferentes formas de prejuicio, discriminación y violencia que los puede hacer vulnerables a las prácticas sexuales de riesgo (7).

Los hallazgos del estudio pueden ser considerados en el diseño de programas tendientes a incrementar el uso de condón entre los HSH. La relación entre actitud hacia el condón con el uso del mismo implica que es necesario mantener e intensificar las campañas de prevención dirigidas a promover la percepción de que el uso del condón puede formar parte de una sexualidad placentera. También 
se requieren medidas para desalentar o reducir el consumo de drogas y alcohol en esta población, ya que se relacionaron con algunas prácticas de riesgo. Para ello, se podría regular la disponibilidad de alcohol en los sitios comerciales donde se reúnen está población (i.e discotecas, bares, etc,). Asimismo, es necesario reducir el estigma asociado con la homosexualidad ya qué se ha observado que la homofobia internalizada se relaciona con mayor consumo de alcohol en la población homosexual y bisexual (29).

Limitaciones del estudio: Se utilizó una muestra por conveniencia de HSH y la información fue captada mediante un cuestionario de auto-respuesta, con lo que es posible que los sujetos modificaran sus respuestas

\section{REFERENCIAS}

1. CENSIDA. EI SIDA en cifras, 2006. México; 2006.

2. CENSIDA. El sida en México. Categorías de Transmisión. México; 31-12-2006.

3. Sistema Estatal de Salud de Chihuahua. Casos acumulados de VIH/SIDA entre 1984 al 2005 en el estado de Chihuahua. México; 2005.

4. Sistema especial de vigilancia epidemiológica de los Servicios de Salud de Chihuahua. Casos nuevos de VIH, Informe del primer semestre. México; 2005.

5. Amuchástegui HA. Aportaciones de las ciencias sociales para la prevención del SIDA. Izazola JA. SIDA: Estado actual del conocimiento. México; 1996: 117-139.

6. Vera Gamboa L, Cerón Bracamonte B, Góngora Biachi R. Hombres que tienen sexo con hombres: conocimientos y prácticas sexuales en relación a la epidemia del VIH/SIDA en Mérida, Yucatán, México. Rev Biomed 2004; 15(3):157-163.

7. Ortiz-Hernández L, Torres MIG. Internalized opression and high-risk sexual practices among homosexual and bisexual males, Mexico. Revista Saude Publica 2005; 39(6):956-964.

8. Izazola-Licea JA, Gortmaker SL, de G, V, Tolbert K, Mann J. Assessment of non-response bias in a probability household survey of male same-gender sexual behavior. Salud Publica Mex 2000; 42(2):90-98.

9. Hernández-Girón C, Cruz-Valdez A, Quiteiro-Trenado M, Peruga A, Hernández-Avila M. Características de comportamiento sexual en hombres de la Ciudad de México. Salud Publica Mex 1999; 41(2):95-100.

10. Nieto-Andrade B, Izazola-Licea JA. Condom use by men with non-stable partners in Mexico City. Salud Publica Mex 1999; 41(2):85-94.

11. Caballero HR, Villasenor SA. Socioeconomic strata as a predictor factor for constant condom use among adolescents. Rev Saude Publica 2001; 35(6):531-538.

12. Caballero-Hoyos R, Villaseñor-Sierra A. Knowledge of HIV/AIDS among urban adolescents: cultural consensus of doubts and uncertainties. Salud Publica Mex 2003; 45 Suppl 1:S108S114.

13. Hernández-Giron CA, Cruz-Valdez A, Quiterio-Trenado M, Uribe-Salas F, Peruga A, HernándezAvila M. Factors associated with condom use in the male population of Mexico City. Int $\mathrm{J}$ STD AIDS 1999; 10(2):112-117.

14. Izazola-Licea JA, Avila-Figueroa RC, Gortmaker SL, Rio-Chiriboga C. The homosexual transmission of HIV/AIDS in Mexico. Salud Publica Mex 1995; 37(6):602-614.

15. Izazola-Licea JA, Valdespino-Gomez JL, Gortmaker SL, Townsend J, Becker J, Palacios-Martinez $M$ et al. HIV-1 seropositivity and behavioral and sociological risks among homosexual and bisexual men in six Mexican cities. J Acquir Immune Defic Syndr 1991; 4(6):614-622. 
16. González Block MA, Liguori AL, Bazua LF. AIDS and social strata in Mexico: the importance of bisexuality. Salud Publica Mex 1990; 32(1):26-37.

17. Gongora-Biachi RA, González-Martínez P. Antibodies against human immunodeficiency virus (HIV) in a population of prostitutes in Merida, Yucatan, Mexico. Rev Invest Clin 1987; 39(3):305-306.

18. Ramírez J, Suarez E, de la RG, Castro MA, Zimmerman MA. AIDS knowledge and sexual behavior among Mexican gay and bisexual men. AIDS Educ Prev 1994; 6(2):163-174.

19. Sánchez-Huesca R, Arellanes-Hernández JL, Pérez-Islas V, Rodríguez-Kuri SE. Estudio de la relación entre consumo de drogas y migración a la frontera norte de México y Estados Unidos. Salud Mental 2006; 29(1):35-43.

20. Guerena-Burgueno F, Benenson AS, Sepulveda-Amor J. HIV-1 prevalence in selected Tijuana sub-populations. Am J Public Health 1991; 81(5):623-625.

21. Izazola-Licea JA, Valdespino-Gomez JL, Sepulveda-Amor J. Risk factors associated with HIV infection in homosexual and bisexual men. Salud Publica Mex 1988; 30(4):555-566.

22. Mansergh G, Naorat S, Jommaroeng R, Jenkins RA, Stall R, Jeeyapant S et al. Inconsistent condom use with steady and casual partners and associated factors among sexuallyactive men who have sex with men in Bangkok, Thailand. AIDS Behav 2006; 10(6):743751.

23. Hays RB, Paul J, Ekstrand M, Kegeles SM, Stall R, Coates TJ. Actual versus perceived HIV status, sexual behaviors and predictors of unprotected sex among young gay and bisexual men who identify as HIV-negative, HIV-positive and untested. AIDS 1997; 11(12):1495-1502.

24. Lemp GF, Hirozawa AM, Givertz D, Nieri GN, Anderson L, Lindegren ML et al. Seroprevalence of HIV and risk behaviors among young homosexual and bisexual men. The San Francisco/ Berkeley Young Men's Survey. JAMA 1994; 272(6):449-454.

25. Gutierrez JP, Molina-Yepez D, Morrison K, Samuels F, Bertozzi SM. Correlates of condom use in a sample of MSM in Ecuador. BMC Public Health 2006; 6:152.

26. Imwin TW, Morgenstern J, Parsons JT, Wainberg M, Labouvie E. Alcohol and sexual HIV risk behavior among problem drinking men who have sex with men: An event level analysis of timeline followback data. AIDS Behav 2006; 10(3):299-307.

27. Remafedi G. Predictors of unprotected intercourse among gay and bisexual youth: knowledge, beliefs, and behavior. Pediatrics 1994; 94(2 Pt 1):163-168.

28. Izazola-Licea JA, Gortmaker SL, de G, V, Tolbert K, Mann J. Sexual behavior patterns and HIV risks in bisexual men compared to exclusively heterosexual and homosexual men. Salud Publica Mex 2003; 45 Supp 5:S662-S671.

29. Ortiz-Hernández L, García Torres MI. Effects of violence and discrimination on the mental health of bisexuals, lesbians, and gays in Mexico City. Cad Saude Publica 2005; 21(3):913-925. 